\title{
Analytical Solutions of the Susceptible-Infected-Virus (SIV) Model
}

\author{
Emily MacIndoe \\ Advisor: Leo Lee \\ University of Mary Washington \\ Author Email: emacindo@mail.uwm.edu \\ Advisor Email: llee3@umw.edu
}

\begin{abstract}
The Susceptible-Infected-Virus (SIV) model is a compartmental model to describe within-host dynamics of a viral infection. We apply the SIV model to the human immunodeficiency virus (HIV); in particular, we present analytical solutions to two versions of the model. The first version includes only terms related to the susceptible cell-virus particle interaction and virus production, while the second includes those terms in addition to the infected cell death rate. An analytical solution, although more challenging and time-consuming than numerical methods, has the advantage of giving exact, rather than approximate, results. These results contribute to our understanding of virus dynamics and could be used to develop better treatment options. The approach used to solve each model involved first isolating one of the dependent variables, that is, deriving an equation that involves only one of the variables and its derivatives. Next, various substitutions were used to bring the equation to a more easily solvable form. For the first model, an exact solution is obtained in the form of an implicit equation. For the second model, we give an analytical solution generated by an iterative method.
\end{abstract}

\section{Introduction}

The Susceptible-Infected-Virus (SIV) model is a system of ordinary differential equations that describes the interaction of virus particles with the cells of a living organism. We apply the SIV model to the human immunodeficiency virus (HIV). To begin, it will be useful to outline the basic concepts of virus-cell dynamics, which are described in more detail in [7]. A virus is essentially genetic material enclosed in a protein shell. The virus particle enters an organism and then infects a cell by attaching to the cell wall and inserting its genetic material, either DNA or RNA. This genetic material makes its way to the cell's nucleus and reprograms the cell to make copies of the virus. After many copies have been made, the cell undergoes either bursting or budding, releasing new virus particles that can go on to infect other cells. In the case of HIV, the new viruses are released through the budding process, so 


\begin{tabular}{|c|c|c|c|}
\hline Parameter & Value & Units & Description \\
\hline$k$ & $1.179 \times 10^{-3}$ & $1 /$ (virions·day) & Infection Rate Constant \\
\hline$\delta$ & 0.3660 & $1 /$ day & Infected Cell Death Rate Constant \\
\hline$N_{T}$ & 4246.4 & virions/cell & $\begin{array}{c}\text { Virus Production Rate Constant } \\
\text { (per infected cell) }\end{array}$ \\
\hline
\end{tabular}

Table 1: Values of Constants (obtained from [7])

that the infected cell remains intact. The cells targeted by HIV are CD4+ T-cells, which are helper cells that aid in immune system responses. In simple terms, HIV impairs the body's ability to fight off viruses and bacteria.

Various HIV models and their properties have been studied. The most common is the basic model of virus dynamics (SIV model), given by

$$
\begin{aligned}
& \frac{d S}{d t}=\lambda-d S-k V S, \\
& \frac{d I}{d t}=k V S-\delta I, \\
& \frac{d V}{d t}=N_{T} \delta I-c V,
\end{aligned}
$$

where $S$ is the number of healthy cells, $I$ is the number of infected cells, and $V$ is the number of free virus particles. The other symbols $\left(\lambda, d, k, \delta, N_{T}\right.$, and $\left.c\right)$ are positive constants. Many researchers have analyzed this model. For example, [1] determines equilibria for the system and analyzes their stability using Lyapunov functions, finding that stability depends on whether the basic reproduction number $R_{0}=k \lambda N_{T} / d c$ is greater than, less than, or equal to unity. Similarly, [7] uses the same model, first showing existence and uniqueness, then analyzing equilibria, and finally reworking the system as a stochastic problem. The article [11] seeks to understand the impact of errors in estimating model parameters; the above model was used, excluding the $\lambda$ and $-d S$ terms in (1.1).

Using (1.1)-(1.3) as a basis, most researchers find that the model as given is too simple for their purposes. Accordingly, more terms must be added to better approximate virus-cell dynamics. Several sources, including [3], [5], [4], [10], and [12], analyze the effects of various treatments on the progression of viruses, with some modelling HIV and others modeling other viruses, such as the Hepatitis B virus (HBV). [10] and [12] also take into account immune system responses. In addition, [12] analyzes the effects of logistic growth, time delays, and infection age on the model. Finally, [2] is interesting in that it replaces the infection rate $k$ above with a generic function $f(V)$ and then analyzes the generalized system.

In each of the sources mentioned above, it should be noted that solutions to the system were obtained from numerical methods; that is, none of the sources attempted to find an exact solution to the system. The goal of this paper is to present analytical solutions to two versions of the model, one including only the interaction and virus production terms, and 
another which introduces a death rate for the infected cells. Our two models are given by

$$
\begin{aligned}
& \frac{d S}{d t}=-k V S \\
& \frac{d I}{d t}=k V S \\
& \frac{d V}{d t}=N_{T} \delta I
\end{aligned}
$$

and

$$
\begin{aligned}
& \frac{d S}{d t}=-k V S, \\
& \frac{d I}{d t}=k V S-\delta I, \\
& \frac{d V}{d t}=N_{T} \delta I,
\end{aligned}
$$

where $\frac{d}{d t}$ indicates differentiation with respect to time. Here $k, N_{T}$, and $\delta$ are positive constants. Values for the constants were obtained from [7] and are given in Table 1. Below we briefly discuss the significance of each equation in the system.

First, examine (1.4). Note that there are no positive terms on the right hand side of the equation, so the number of susceptible cells must always be decreasing. The term $k V S$ is the infection rate, or the rate at which healthy susceptible cells are converted into infected cells. This rate is proportional to the product of the virus particles and susceptible cells $V S$, with proportionality constant $k$. Next, examining (1.5), we see that $d I / d t$ is always positive, so that the number of infected cells is increasing. In fact, $I$ increases at the same rate that $S$ decreases. Finally, note (1.6). The number of free virus particles $V$ increases at a rate proportional to the number of infected cells $I$, with proportionality constant $N_{T} \delta$. The second system is nearly identical to the first. The only difference here is that we have added in the term $-\delta I$, representing the infected cell death rate. Note that, since HIV-infected cells undergo the budding process, $-\delta I$ does not represent the rate at which infected cells are destroyed by virus particles, as one might be tempted to assume. Instead, the infected cells are left in tact after virus particles are released, which is why there is no problem with excluding this term from the first model.

These systems should show the same general trend as the full system early on in the course of the disease; namely, $S$ decreases in time and $I$ and $V$ increase in time. Comparison of the three systems using numerical methods reveals that the approximations are not particularly accurate; nonetheless, solving the simplified models is a useful exercise and provides insight into the interactions of terms in the system. 


\section{Solution of the First Model}

Recall that our first version of the SIV model is given by

$$
\begin{aligned}
& \frac{d S}{d t}=-k V S, \\
& \frac{d I}{d t}=k V S, \\
& \frac{d V}{d t}=N_{T} \delta I .
\end{aligned}
$$

Before attempting to solve this system, we take a moment to verify that a unique solution exists. To do so, we use the Picard-Lindelöf Theorem, which is given in [8].

Theorem 2.1 (Picard-Lindelöf Theorem). Let $n \in \mathbb{N}$ and $y_{0} \in \mathbb{R}^{n}$ be given. Assume the function $f: \mathbb{R}^{n} \times \mathbb{R} \rightarrow \mathbb{R}^{n}$ is locally Lipschitz in its first argument and continuous in its second argument. Then there exist $t^{*}>0$ and a unique function $y:\left[0, t^{*}\right] \rightarrow \mathbb{R}^{n}$ satisfying

$$
y^{\prime}(t)=f(y(t), t)
$$

for every $t \in\left[0, t^{*}\right]$ and the initial condition $y(0)=y_{0}$.

For our system,

$$
y=\left[\begin{array}{c}
S \\
I \\
V
\end{array}\right], \quad f(y)=\left[\begin{array}{c}
-k V S \\
k V S \\
N_{T} \delta I
\end{array}\right],
$$

where the system is said to be autonomous since it does not explicitly depend on the independent variable $t$. Computing the Jacobian matrix gives

$$
J=\left[\begin{array}{ccc}
-k V & 0 & -k S \\
k V & 0 & k S \\
0 & N_{T} \delta & 0
\end{array}\right],
$$

from which we see that the partial derivatives of $f$ exist and are continuous for any values of $S, I, V$, and $t$. It follows that $f$ is Lipschitz continuous, and hence a unique solution exists on some interval $\left[0, t^{*}\right]$.

Having verified the existence of a unique solution, we are now ready to solve the system. First note

$$
\frac{d}{d t}(S+I)=0,
$$

which implies

$$
S+I=P,
$$

where $P$ is a constant determined by the initial conditions. It follows that

$$
\frac{d V}{d t}=N_{T} \delta P-N_{T} \delta S .
$$


Applying the chain rule,

$$
\frac{d S}{d V}=\frac{d S / d t}{d V / d t}=-\frac{k}{N_{T} \delta} V \frac{S}{P-S},
$$

separating variables and integrating,

$$
P \ln |S|-S=-\frac{k}{2 N_{T} \delta} V^{2}+D .
$$

Solving for $V$ gives

$$
V= \pm \sqrt{C-\frac{2 N_{T} \delta P}{k} \ln |S|+\frac{2 N_{T} \delta}{k} S} .
$$

Here, $C$ and $D$ are constants determined by the initial conditions and related by $C=$ $2 N_{T} \delta D / k$. To simplify, we assume that $S$ and $V$ are non-negative, yielding

$$
V=\sqrt{C-\frac{2 N_{T} \delta P}{k} \ln S+\frac{2 N_{T} \delta}{k} S} .
$$

Substituting this expression for $V$ into the derivative for $S$ gives

$$
\frac{d S}{d t}=-k\left(\sqrt{C-\frac{2 N_{T} \delta P}{k} \ln S+\frac{2 N_{T} \delta}{k} S}\right) S .
$$

Now, separating variables and integrating,

$$
\int_{S_{0}}^{S} \frac{1}{\xi}\left(C-\frac{2 N_{T} \delta P}{k} \ln \xi+\frac{2 N_{T} \delta}{k} \xi\right)^{-1 / 2} d \xi=-k \int_{t_{0}}^{t} d \xi=-k t,
$$

where we have set $t_{0}=0$. The integral above implicitly gives $S$ as a function of $t$. $I$ and $V$ are then found from Equations (2.4) and (2.5), respectively. Under the initial conditions

$$
S(0)=S_{0}, \quad I(0)=I_{0}, \quad V(0)=V_{0},
$$

the constants $P$ and $C$ are

$$
P=S_{0}+I_{0} \quad \text { and } \quad C=V_{0}^{2}+\frac{2 N_{T} \delta P}{k} \ln S_{0}-\frac{2 N_{T} \delta}{k} S_{0} .
$$



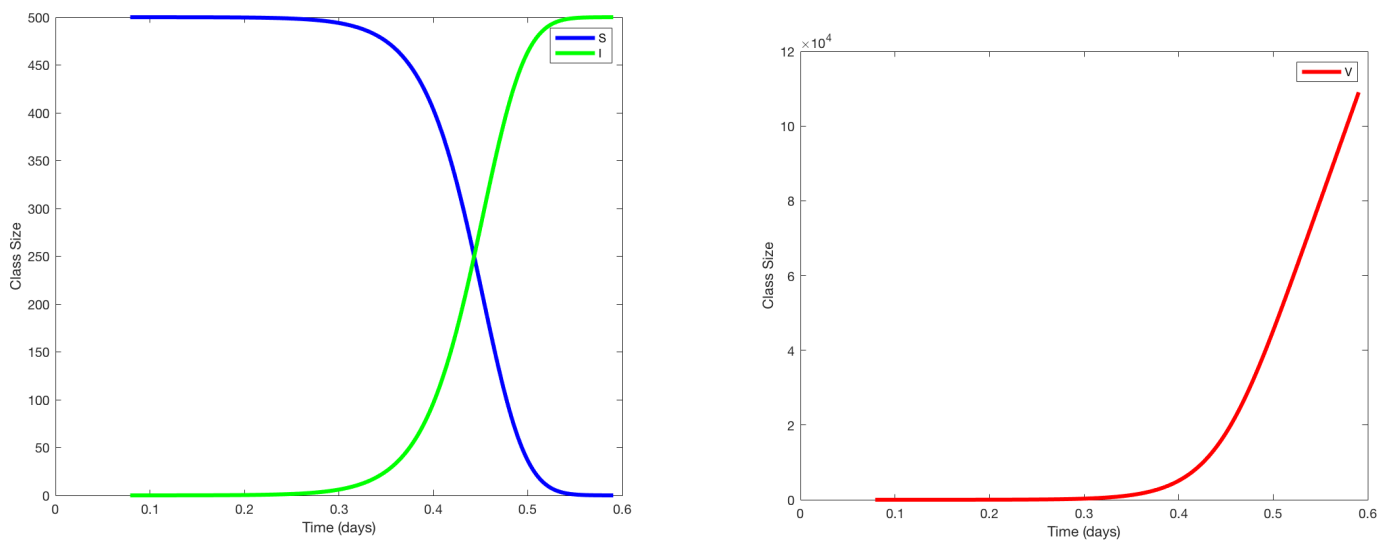

Figure 1: Plot of $S$ and $I$ (left, in number of cells per cubic milliliter) and $V$ (right, in number of free virus particles per cubic millimeter) over time (in days).

Figure 1, created in MATLAB, gives plots of the solution for the initial conditions $S(0)=500, I(0)=0$, and $V(0)=0.1$. The plots were generated using the implicit analytical solution, under the assumption that $S$ strictly decreases from its starting value to its equilibrium value.

\section{Solution of the Second Model}

Next, we introduce the $\delta I$ term in Equation (2.2), giving our second version of the SIV model,

$$
\begin{aligned}
& \frac{d S}{d t}=-k V S, \\
& \frac{d I}{d t}=k V S-\delta I, \\
& \frac{d V}{d t}=N_{T} \delta I,
\end{aligned}
$$

with the initial conditions

$$
S(0)=S_{0}, \quad I(0)=I_{0}, \quad V(0)=V_{0} .
$$

Once again, we start by verifying the existence and uniqueness of a solution. Computing the Jacobian matrix for the second system gives

$$
J=\left[\begin{array}{ccc}
-k V & 0 & -k S \\
k V & -\delta & k S \\
0 & N_{T} \delta & 0
\end{array}\right] .
$$

As before, the partial derivatives exist and are continuous for any $S, I, V$, and $t$. Therefore, a unique solution exists on $\left[0, t^{*}\right]$ for some $t^{*} \in \mathbb{R}$. 
Having verified existence and uniqueness, we are now ready to solve the system. Firstly, note that

$$
\frac{d}{d t}\left(S+I+\frac{V}{N_{T}}\right)=0
$$

which implies

$$
S+I+\frac{V}{N_{T}}=P,
$$

where $P$ is a constant determined by the initial conditions. In fact,

$$
P=S_{0}+I_{0}+\frac{V_{0}}{N_{T}} .
$$

Now, from Equation (3.4), we find

$$
I=P-S-\frac{V}{N_{T}}
$$

and

$$
S=P-I-\frac{V}{N_{T}} .
$$

Substituting for $I$ in Equation (3.3), differentiating, and applying (3.5) and (3.6) gives

$$
\frac{d^{2} V}{d t^{2}}=N_{T} \delta k P V-k V \frac{d V}{d t}-\delta k V^{2}-\delta \frac{d V}{d t} .
$$

Thus, we have reduced the system to a single differential equation,

$$
V^{\prime \prime}=N_{T} \delta k P V-k V V^{\prime}-\delta k V^{2}-\delta V^{\prime},
$$

where the prime indicates differentiation with respect to $t$. We next use a series of substitutions to bring (3.7) to a more easily solvable form. Using a transformation from [9], let

$$
u=\frac{d t}{d V}=\frac{1}{V^{\prime}},
$$

with the initial condition

$$
u\left(V_{0}\right)=\frac{1}{N_{T} \delta I_{0}} .
$$

Then we find

$$
\frac{d u}{d V}=\left(\delta k V^{2}-N_{T} \delta k P V\right) u^{3}+(k V+\delta) u^{2} .
$$


Equation (3.9) is an Abel equation of the first kind; that is, it has the form

$$
\frac{d u}{d V}=f_{3}(V) u^{3}+f_{2}(V) u^{2}+f_{1}(V) u+f_{0}(V),
$$

where in this case

$$
\begin{aligned}
& f_{3}(V)=\delta k V^{2}-N_{T} \delta k P V \\
& f_{2}(V)=k V+\delta \\
& f_{1}(V)=0 \\
& f_{0}(V)=0 .
\end{aligned}
$$

In [9], an iterative method is used to solve an equation of this type. Essentially, we obtain a sequence of approximate analytical solutions to the equation. The exact solution is given by the limit of the sequence at infinity. To start, let

$$
\phi=\ln u,
$$

or in other words,

$$
u=e^{\phi} .
$$

We then have the initial condition

$$
\phi\left(V_{0}\right)=-\ln \left(N_{T} \delta I_{0}\right) .
$$

The differential equation then becomes

$$
\frac{d \phi}{d V}=\left(\delta k V^{2}-N_{T} \delta k P V\right) e^{2 \phi}+(k V+\delta) e^{\phi} .
$$

The expression $e^{\phi}$ can be expanded in a Taylor series as

$$
e^{\phi}=\sum_{n=0}^{\infty} \frac{\phi^{n}}{n !}=1+\phi+\frac{\phi^{2}}{2}+\frac{\phi^{3}}{6}+\cdots,
$$

so we may write (3.13) as

$$
\begin{aligned}
\frac{d \phi}{d V}= & \left(\delta k V^{2}-N_{T} \delta k P V\right)\left(1+2 \phi+\frac{(2 \phi)^{2}}{2}+\cdots\right) \\
& +(k V+\delta)\left(1+\phi+\frac{\phi^{2}}{2}+\cdots\right) .
\end{aligned}
$$

As a first approximation, $\phi_{1}(V)$, use only the first two terms of the Taylor series; that is, let

$$
\begin{aligned}
\frac{d \phi_{1}}{d V} & =\left(\delta k V^{2}-N_{T} \delta k P V\right)\left(1+2 \phi_{1}\right)+(k V+\delta)\left(1+\phi_{1}\right) \\
& =\left[\delta k V^{2}+\left(k-N_{T} \delta k P\right) V+\delta\right]+\left[2 \delta k V^{2}+\left(k-2 N_{T} \delta k P\right) V+\delta\right] \phi_{1} .
\end{aligned}
$$


The above is a first order, linear differential equation and can be solved with an integrating factor. Let

$$
F=\exp \left[-\frac{2}{3} \delta k V^{3}-\left(\frac{k}{2}-N_{T} \delta k P\right) V^{2}-\delta V\right]
$$

Then

$$
\phi_{1}(V)=\frac{1}{F(V)}\left\{-\ln \left(N_{T} \delta I_{0}\right) F\left(V_{0}\right)+\int_{V_{0}}^{V} F(\xi)\left[\delta k \xi^{2}+\left(k-N_{T} \delta k P\right) \xi+\delta\right] d \xi\right\},
$$

where we have taken $\phi_{1}\left(V_{0}\right)=\phi\left(V_{0}\right)$. Note that (3.14) can also be written as

$$
\begin{aligned}
\frac{d \phi}{d V}= & \left(\delta k V^{2}-N_{T} \delta k P V\right)\left[1+2 \phi+\sum_{n=2}^{\infty} \frac{(2 \phi)^{n}}{n !}\right] \\
& +(k V+\delta)\left[1+\phi+\sum_{n=2}^{\infty} \frac{\phi^{n}}{n !}\right],
\end{aligned}
$$

or rearranging,

$$
\begin{aligned}
\frac{d \phi}{d V}= & {\left[2 \delta k V^{2}+\left(k-2 N_{T} \delta k P\right) V+\delta\right] \phi+\left[\delta k V^{2}+\left(k-N_{T} \delta k P\right) V+\delta\right] } \\
& +\sum_{n=2}^{\infty}\left[\left(\delta k V^{2}-N_{T} \delta k P V\right) \frac{(2 \phi)^{n}}{n !}+(k V+\delta) \frac{\phi^{n}}{n !}\right] .
\end{aligned}
$$

For a second approximation, $\phi_{2}(V)$, plug into the infinite sum the expression for $\phi_{1}$ obtained previously; that is, let

$$
\begin{aligned}
\frac{d \phi_{2}}{d V}= & {\left[2 \delta k V^{2}+\left(k-2 N_{T} \delta k P\right) V+\delta\right] \phi_{2}+\left[\delta k V^{2}+\left(k-N_{T} \delta k P\right) V+\delta\right] } \\
& +\sum_{n=2}^{\infty}\left[\left(\delta k V^{2}-N_{T} \delta k P V\right) \frac{\left(2 \phi_{1}\right)^{n}}{n !}+(k V+\delta) \frac{\phi_{1}^{n}}{n !}\right] .
\end{aligned}
$$

Just as before, this is a first order, linear equation and can be solved with an integrating factor. The coefficient of $\phi_{2}$ is the same as the coefficient of $\phi_{1}$ in Equation (3.15), so we may use the same integrating factor. Thus,

$$
\begin{aligned}
\phi_{2}(V)= & -\frac{1}{F(V)} \ln \left(N_{T} \delta I_{0}\right) F\left(V_{0}\right)+\frac{1}{F(V)} \int_{V_{0}}^{V} F(\xi)\left[\delta k \xi^{2}+\left(k-N_{T} \delta k P\right) \xi+\delta\right] d \xi \\
& +\frac{1}{F(V)} \int_{V_{0}}^{V} F(\xi) \sum_{n=2}^{\infty}\left\{\left(\delta k \xi^{2}-N_{T} \delta k P \xi\right) \frac{\left[2 \phi_{1}(\xi)\right]^{n}}{n !}+(k \xi+\delta) \frac{\left[\phi_{1}(\xi)\right]^{n}}{n !}\right\} d \xi,
\end{aligned}
$$

where, as before, we have taken $\phi_{2}\left(V_{0}\right)=\phi\left(V_{0}\right)$. Continuing this process gives successively more accurate estimates for $\phi(V)$. In general, for any integer $m \geq 1$,

$$
\begin{gathered}
\phi_{m+1}(V)=\frac{-1}{F(V)} \ln \left(N_{T} \delta I_{0}\right) F\left(V_{0}\right)+\frac{1}{F(V)} \int_{V_{0}}^{V} F(\xi)\left[\delta k \xi^{2}+\left(k-N_{T} \delta k P\right) \xi+\delta\right] d \xi \\
+\frac{1}{F(V)} \int_{V_{0}}^{V} F(\xi) \sum_{n=2}^{\infty}\left\{\left(\delta k \xi^{2}-N_{T} \delta k P \xi\right) \frac{\left[2 \phi_{m}(\xi)\right]^{n}}{n !}+(k \xi+\delta) \frac{\left[\phi_{m}(\xi)\right]^{n}}{n !}\right\} d \xi,
\end{gathered}
$$


or in a more compact form,

$$
\begin{aligned}
\phi_{m+1}(V)=\phi_{1}(V) & +\frac{1}{F(V)} \int_{V_{0}}^{V} F(\xi)\left(\delta k \xi^{2}-N_{T} \delta k P \xi\right)\left[e^{2 \phi_{m}(\xi)}-1-2 \phi_{m}(\xi)\right] d \xi \\
& +\frac{1}{F(V)} \int_{V_{0}}^{V} F(\xi)(k \xi+\delta)\left[e^{\phi_{m}(\xi)}-1-\phi_{m}(\xi)\right] d \xi
\end{aligned}
$$

In light of (3.16), this simplifies to

$$
\begin{aligned}
\phi_{m+1}(V)= & -\frac{\ln \left(N_{T} \delta I_{0}\right) F\left(V_{0}\right)}{F(V)}+\frac{\delta k}{F(V)} \int_{V_{0}}^{V} F(\xi)\left(\xi^{2}-N_{T} P \xi\right)\left[e^{2 \phi_{m}(\xi)}-2 \phi_{m}(\xi)\right] d \xi \\
& +\frac{1}{F(V)} \int_{V_{0}}^{V} F(\xi)(k \xi+\delta)\left[e^{\phi_{m}(\xi)}-\phi_{m}(\xi)\right] d \xi .
\end{aligned}
$$

With each iteration, the approximation $\phi_{m}(V)$ should become closer to $\phi(V)$, the solution of (3.13); that is, $\lim _{m \rightarrow \infty} \phi_{m}(V)=\phi(V)$. Having outlined a method to obtain $\phi$, we may now work backwards to find $V(t)$. By (3.12) and (3.8), we have

$$
\frac{d t}{d V}=e^{\phi(V)}
$$

or integrating both sides,

$$
\int_{t_{0}}^{t} d \xi=\int_{V_{0}}^{V} e^{\phi(\xi)} d \xi
$$

Taking $t_{0}=0, V(t)$ is given implicitly by

$$
t=\int_{V_{0}}^{V} e^{\phi(\xi)} d \xi
$$

Now, by Equation (3.1),

$$
\frac{d S}{d t}=-k V S
$$

Separating variables and integrating,

$$
\int_{S_{0}}^{S} \frac{1}{\xi} d \xi=-k \int_{t_{0}}^{t} V(\xi) d \xi
$$

which implies (for $t_{0}=0$ )

$$
S=S_{0} \exp \left[-k \int_{0}^{t} V(\xi) d \xi\right]
$$

Finally, by (3.5),

$$
I=P-S_{0} \exp \left[-k \int_{0}^{t} V(\xi) d \xi\right]-\frac{V(t)}{N_{T}} .
$$


This completes the analytical solution of the second simplified model, with $V$ given implicitly by (3.18) and $S$ and $I$ given in terms of $V$ by (3.19) and (3.20), respectively.

Figure 2 shows several approximations for $\phi, V, S$, and $I$, obtained by iterating 1200, 2400, 3600, 4800, and 6000 times. The initial conditions $S(0)=500, I(0)=0.1$, and $V(0)=0.1$ were used. Due to the complexity of the analytical solution, the calculations were quite time-consuming; accordingly, the solutions were only computed on a small interval. The curves for $\phi$ and $V$ have the distinctive behavior of coinciding almost perfectly with the exact solution (in light blue) at first and then abruptly branching off; with each successive approximation, the branching point moves farther to the right. To analyze the system's behavior on a larger interval, Euler's method was used to generate Figure 3.
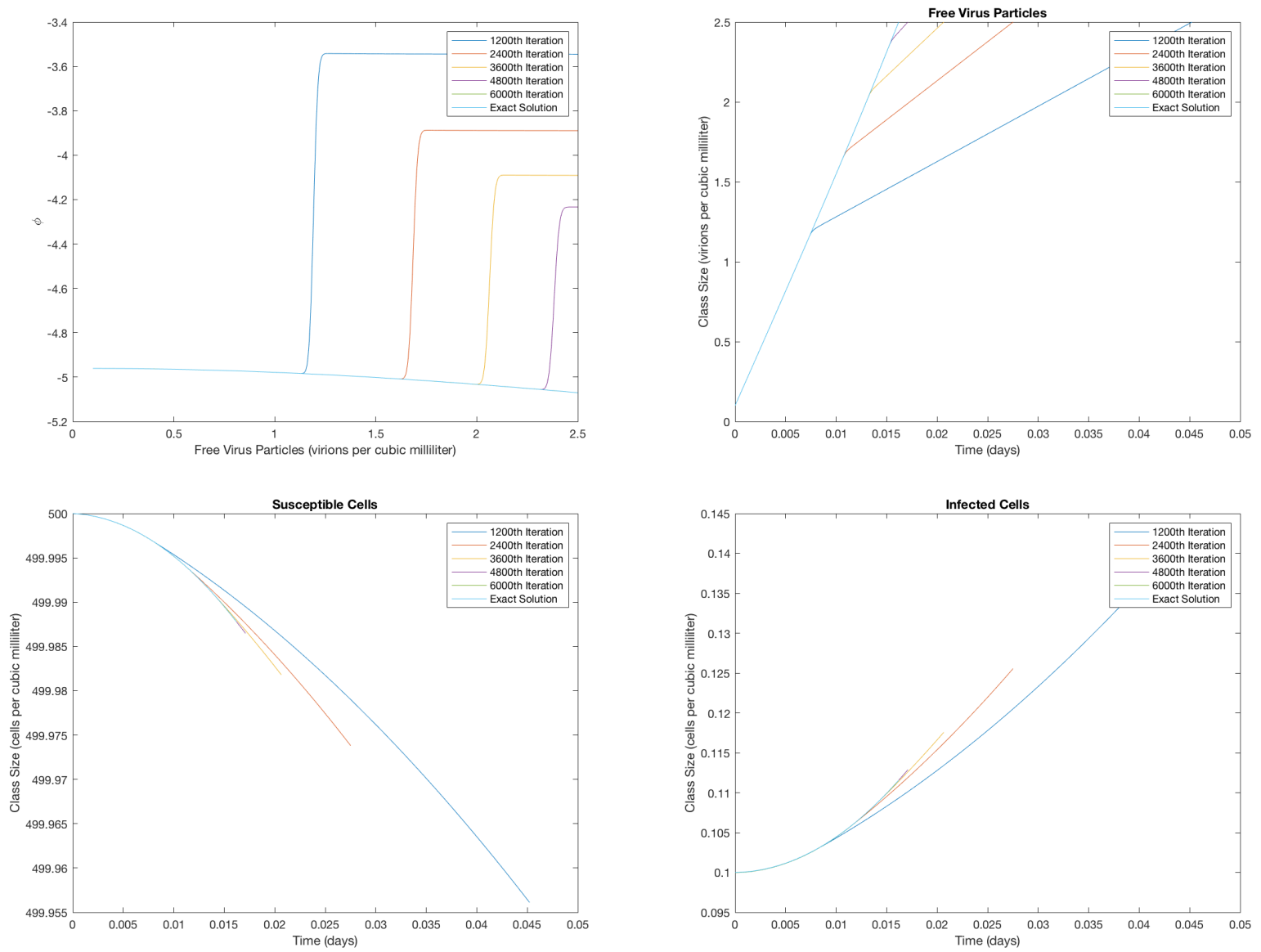

Figure 2: Plots for $\phi$ (top left), $V$ (top right), $S$ (bottom left), and $I$ (bottom right) using the analytical solution. The dark blue, orange, yellow, purple, and green curves show the approximations obtained from $\phi_{1201}, \phi_{2401}, \phi_{3601}, \phi_{4801}$, and $\phi_{6001}$, respectively. The light blue curve represents the exact solution (computed from Euler's method). Note that the green and light blue curves overlap. 

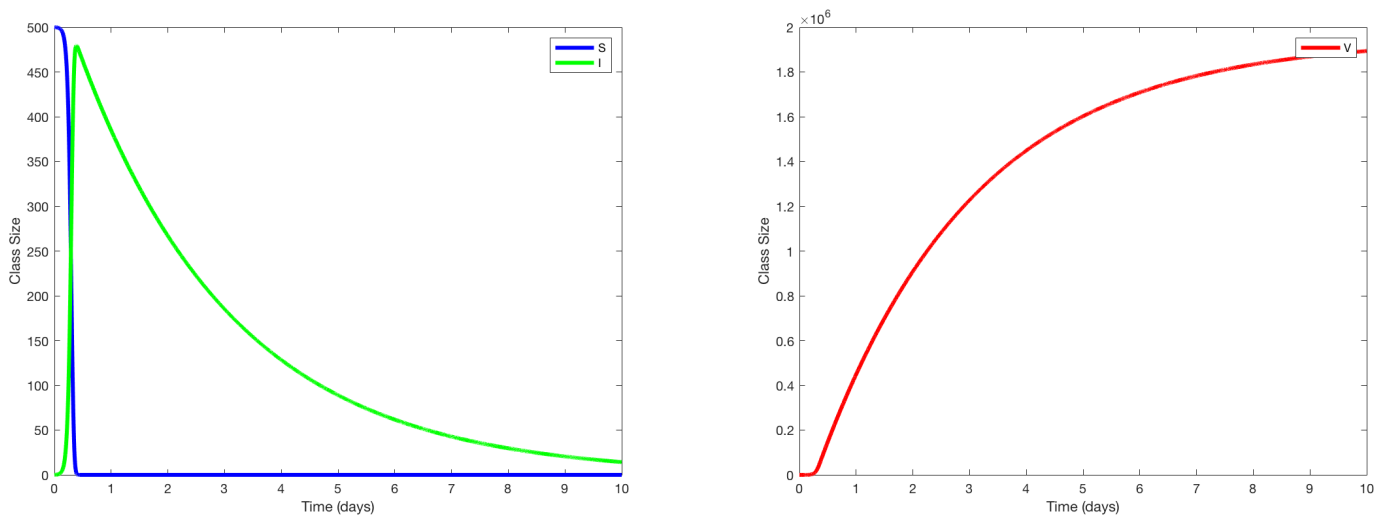

Figure 3: Plot of $S$ and $I$ (left, in number of cells per cubic milliliter) and $V$ (right, in number of virus particles per cubic milliliters) over time (in days) for the second model.

\section{Discussion}

It is interesting to compare the results of the two models. Examining Figures 1 and 3, we see that the models start out similarly, with a level period followed by a sharp decrease in $S$ and with sharp increases in $I$ and $V$. One noticeable difference is that $I$ experiences more moderate growth in the second model, which is to be expected since we have incorporated its death rate. The same can be said for $V$, whose increase depends on $I$. The long term behaviors of the models, on the other hand, are quite different. In both models, $S$ eventually reaches the same equilibrium value of zero. Whereas in the first model, $I$ eventually leveled off to a positive value (the same as the starting value for $S$ ), in the second, $I$ eventually drops to zero. In the second model, rather than continuing to grow linearly as it does in the first, $V$ eventually reaches an equilibrium value. This occurs because there are no more infected cells to produce virus particles. To extend on this work, it would be interesting to next incorporate the viral clearance rate. This should give more realistic short term and long term outputs. On the whole, our two models give a great deal of insight into the roles of the infection term, virus production term, and infected cell death rate in the SIV model.

\section{References}

[1] A. KOROBEINIKOV, Global properties of basic virus dynamics models, Bull. Math. Biol., 66 (2004) pp. 879-883; also available online from https://link.springer.com/article/10.1016\%2Fj.bulm.2004.02.001.

[2] C. DAI, C. MA, L. SONG, AND K. WANG, Dynamics of a Viral Infection Model with General Contact Rate between Susceptible Cells and Virus Particles, Discrete Dyn. Nat. Soc., (2014); also available online from https://www.hindawi.com/journals/aaa/2014/546795/. 
[3] D. WODARZ AND M. A. NOWAK, Mathematical models of HIV pathogenesis and treatment, BioEssays, 24 (2002), pp. 1178-1187; also available online from http://ped.fas.harvard.edu/files/ped/files/bioessays02_0.pdf.

[4] J. YANG, X. WANG, AND F. ZHANG, A Differential Equation Model of HIV Infection CD 4+ T-Cells with Delay, Discrete Dyn. Nat. Soc., (2008); also available online from https://www.hindawi.com/journals/ddns/2008/903678/.

[5] K. HATTAF, M. RACHIK, S. SAADI, AND N. YOUSIF, Optimal Control of Treatment in a Basic Virus Infection Model, Appl. Math. Sci., 3 (2009), pp. 949-958; also available online from https://www.mapleprimes.com/DocumentFiles/205660_Answer/ Optimal_Control_of_T.pdf.

[6] L. MIN, Y. SU, AND Y. KUANG, Mathematical Analysis of a Basic Virus Infection Model with Application to HBV Infection, Rocky Mountain J. Math., 38 (2008), pp. 1573-1585; also available online from https://math.la.asu.edu/ kuang/paper/MinSuKuang.pdf.

[7] P. A. ROMER, Stochastic Modeling of the Persistence of HIV: Early Population Dynamics, United States Naval Academy - Trident Scholar project report, no. 420, 2013.

[8] R. G. BARTLE, Introduction to Real Analysis, Wiley, Dordrecht, 2000.

[9] T. HARKO, F. S. N. LOBO, AND M. K. MAK, Exact analytical solutions of the Susceptible-Infected-Recovered (SIR) epidemic model and of the SIR model with equal death and birth rates, Appl. Math. Comput., 236 (2014), pp. 184-194; also available online from https://arxiv.org/pdf/1403.2160.pdf.

[10] T. S. JANG, J. KIM, H. D. KWON, AND J. Lee, Hybrid On-Off Controls for an HIV Model Based on a Linear Control Problem, J. Korean Math. Soc., 52 (2015), pp. 469-487; also available online from http://www.mathnet.or.kr/mathnet/thesis_file/JKMS-52-3-469-487.pdf.

[11] V. N. NGUYEN, F. KLAWONN, R. MIKOLAJCZYK, AND E. A. HERNANDEZ-VARGAS, Analysis of Practical Identifiability of a Viral Infection Model, PLoS ONE, 11 (2016), https://journals.plos.org/plosone/article?id=10.1371/journal.pone.0167568.

[12] X. LAI, Study of Virus Dynamics by Mathematical Models, Ph.D. thesis, The University of Western Ontario, London, Ontario, Canada, 2014. 